Bulletin of the Seismological Society of America. Vol. 64, No. 5, pp. 1389-1403. October 1974

\title{
SURFACE MOTION OF A SEMI-ELLIPTICAL ALLUVIAL VALLEY FOR INCIDENT PLANE $S H$ WAVES
}

\author{
By H. L. Wong and M. D. Trifunac
}

\begin{abstract}
By using the exact analytical solution for the two-dimensional $\mathrm{SH}$-wave propagation in and around an elastic inclusion whose cross section corresponds to one half of an ellipse, we have examined those aspects of the resulting ground motion that are of special interest for earthquake engineering and strong-motion seismology. Computed amplitudes and phases of periodic ground motion display complicated wave-interference phenomena that lead to nearly-standing wave patterns, abrupt changes in the amplification of incident motions along the free surface of the alluvial valley and strong dependence of the overall motions on the incidence angle of $S H$ waves. By comparing the amplification patterns derived from the exact model with the amplifications computed on the basis of an equivalent single-layer model excited by the vertically incident shear waves, we have demonstrated that this approximate representation may lead to meaningful results only if the wavelength of incident waves is longer than the characteristic dimension of the alluvial valley. Although simple, we expect that the model presented in this paper might explain qualitatively the vibrations of some alluvial valleys excited by $S H$ components of strong ground motion.
\end{abstract}

\section{INTRODUCTION}

Field observations following large earthquakes have indicated that the areas of intense damage may be highly localized (e.g., Jennings, 1971; Sozen et al., 1968; Hudson, 1972; Gutenberg, 1957; Richter, 1958). Although numerous factors, such as the overall quality of construction and the poor soil conditions, may have contributed significantly to such observations, it has been suggested frequently that one of the principal causes for the observed distributions of damage should be attributed to the amplification of incident waves by the local geological and surface soil conditions. Consequently, in earthquake engineering and strong-motion seismology research, significant effort is now being directed toward better understanding of these amplification phenomena (e.g., Tsai, 1969; Aki and Larner, 1970; Boore, 1970; Trifunac, 1971). To this end, we investigate in this paper the amplification patterns and other related phenomena associated with the plane $\mathrm{SH}$ wave motion in and around the semi-elliptical alluvial valley. Although there might be only a few known alluvial valleys with a cross section that resembles one half of an ellipse (Trifunac, 1971), the exact nature of the solution presented in this paper enables us to understand and explain many important properties of the two-dimensional wave propagation in similar geometries. Furthermore, we expect that the exact solution presented in this paper for the elastic unattenuated $S H$ waves should be of some use for critical calibration and checking of the approximate finite element (e.g., Dezfulian and Seed, 1969) and finite difference calculations (e.g., Boore et al., 1971) which were recently developed for the related wave-propagation problems. 


\section{THE MODEL}

The model to be analyzed in this paper is shown in Figure 1. It consists of a semielliptical elastic inclusion for $\xi \leqq \xi_{0}$ and the elastic isotropic and homogeneous halfspace. To facilitate the analysis, we define the half-space by $y \leqq 0$ for the shallow elliptical inclusion and by $x \geqq 0$ for the deep elliptical inclusion, the focal length of the elliptical coordinate system being $a$ in both cases. The contact between the inclusion and the halfspace is assumed to be welded. The material properties in the half-space are given by the rigidity $\mu$ and the velocity of shear waves $\beta$. To designate these and other quantities for $\xi \leqq \xi_{0}$, we will employ the subscript or superscript $v$.

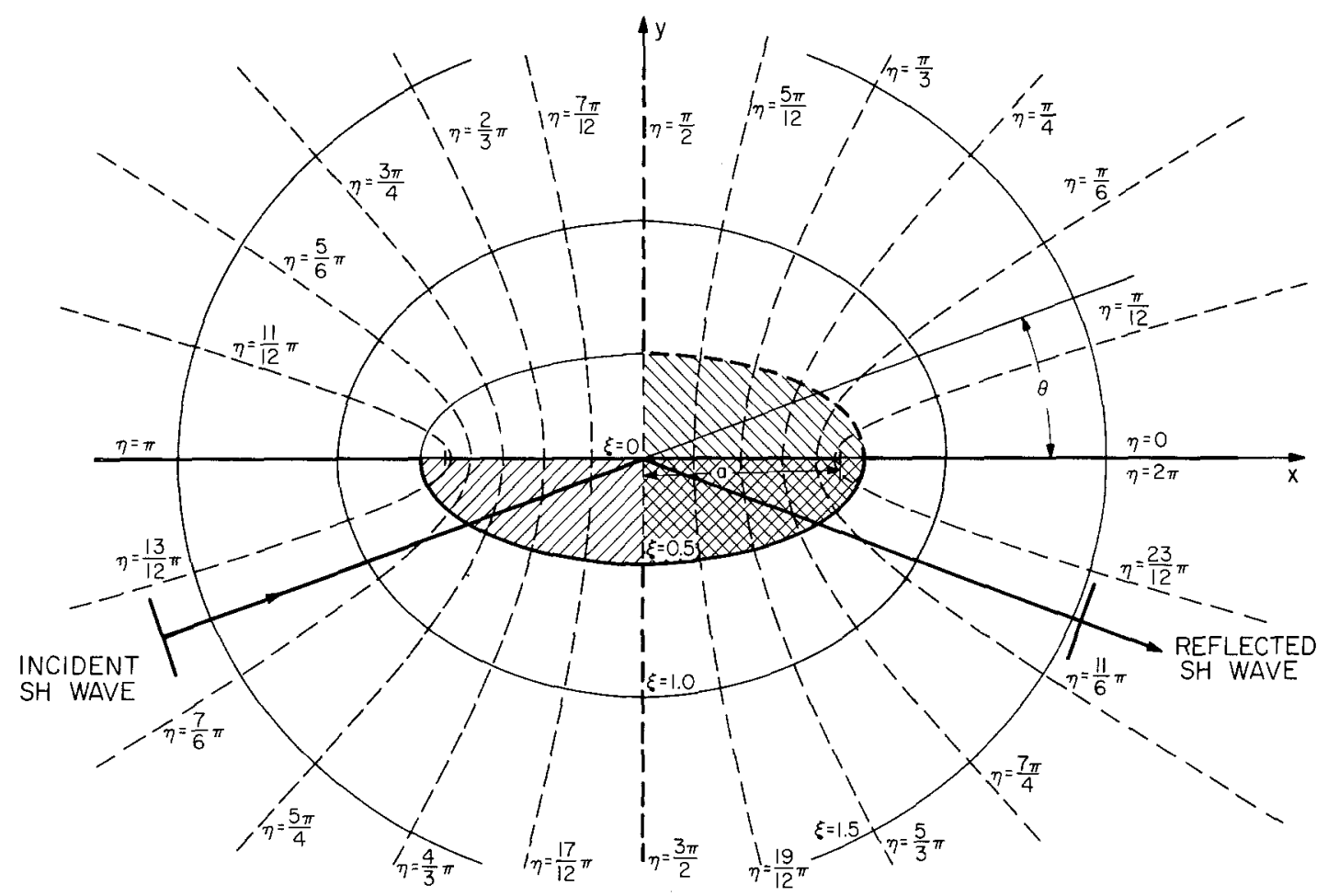

FIG. 1. Shallow and deep semi-elliptical alluvial valleys and the coordinate systems used.

The excitation of the half-space, $u_{z}{ }^{i}$, will consist of an infinite train of plane $S H$ waves with frequency $\omega$ and the nonzero motion in the $z$ direction only

$$
u_{z}^{i}=\exp \left\{-i \omega\left(t-x / c_{x}-y / c_{y}\right)\right\},
$$

where $c_{x}=\beta / \cos \theta$ and $c_{y}=\beta / \sin \theta$ are the phase velocities along the $x$ and $y$ coordinates. Far from the inclusion, the $u_{z}^{r}$ waves reflect from the free surface, $y=0$, interfere with the incident waves, $u_{z}{ }^{i}$, and give

$$
u_{z}^{i+r} \equiv u_{z}^{i}+u_{z}^{r}=2 \exp \left[-i \omega\left(t-x / c_{x}\right)\right] \cos \left(\omega y / c_{y}\right)
$$

\section{Solution of the Problem}

\section{The Elliptical Coordinates}

It is, of course, convenient to use the elliptical coordinate system to solve the boundary value problems for media with boundaries described by ellipses and hyperbolas. 
The elliptical coordinates are the radial coordinate, $\xi$, and the angular coordinate, $\eta$. They are related to the rectangular $x-y$ coordinate system by

$$
\begin{array}{ll}
x=a \cosh \xi \cos \eta & 0<\xi<\infty \\
y=a \sinh \xi \sin \eta & 0<\eta<2 \pi .
\end{array}
$$

As $\xi \rightarrow 0$, the ellipse reduces to a line, and for $\xi \rightarrow \infty$, the ellipse tends to a circle.

The minor-to-major axis ratio for the ellipse with $\xi=\xi_{0}$ is

$$
R \equiv \frac{\operatorname{minor} \text { axis }}{\text { major axis }}=\tanh \xi_{0}
$$

where

$$
\begin{aligned}
& \text { major axis length }=a \cosh \xi_{0} \\
& \text { minor axis length }=a \sinh \xi_{0}
\end{aligned}
$$

and $a$ is the focal length.

From (1.1) and (1.2) one may solve for $\eta$ and $\xi$ in terms of $x$ and $y$ simultaneously to get

$$
\begin{aligned}
& \left(\frac{x}{a \cosh \xi}\right)^{2}+\left(\frac{y}{a \sinh \xi}\right)^{2}=1 \\
& \left(\frac{x}{a \cos \eta}\right)^{2}-\left(\frac{y}{a \sin \eta}\right)^{2}=1
\end{aligned}
$$

an ellipse for $\xi$ and a hyperbola for $\eta$.

\section{The Wave Equation}

One can show that the harmonic wave equation has the time-independent part satisfying the Helmholtz equation

where

$$
\frac{\partial^{2} \psi}{\partial \xi^{2}}+\frac{\partial^{2} \psi}{\partial \eta^{2}}+k^{2} \psi=0
$$

$$
k=\omega / \beta \text {. }
$$

Using equations (1.1) and (1.2) we can transform (2.1) to elliptical coordinates

$$
\frac{\partial^{2} \psi}{\partial \xi^{2}}+\frac{\partial^{2} \psi}{\partial \eta^{2}}+a^{2} k^{2}\left[\cosh ^{2} \xi-\cos ^{2} \eta\right] \psi=0 .
$$

The most general solution of equation (2.1) (Morse and Feshbach, 1953) is of the form

$$
\psi(x, y, t)=\int_{0}^{2 \pi} F[x \cos u+y \sin u-c t] d u .
$$

However, if the boundary is a rectangle, a circle, an ellipse, or a parabola, the integral (2.4) may degenerate to an infinite series of the form (Mow and Pao, 1971)

$$
\sum_{m=0}^{\infty} a_{m} Z_{m}(\xi) H_{m}(\eta)
$$

The functions $Z_{m}$ and $H_{m}$ are then solutions of the separate ordinary differential equations in $\xi$ and $\eta$. 
For the elliptical coordinates the functions $Z_{m}$ and $H_{m}$ are the solutions of the ordinary differential equations

$$
\begin{aligned}
& \frac{d^{2} H}{d \eta^{2}}+(b-2 q \cos 2 \eta) H=0 \\
& \frac{d^{2} Z}{d \xi^{2}}-(b-2 q \cosh 2 \xi) Z=0
\end{aligned}
$$

where

$$
q=\frac{1}{4} a^{2} k^{2}=\frac{1}{4} \frac{a^{2} \omega^{2}}{\beta^{2}} .
$$

Equation (2.5) is called the Mathieu's equation, while (2.6) is called the modified Mathieu equation because it reduces to $(2.5)$ if $\xi$ is replaced by $-i \eta$.

We shall look for periodic solutions of (2.5) only, since $\psi$ must be the same at $\eta=0$, $\eta=2 n \pi$, etc. These periodic solutions exist only for a countable number of discrete values of $b$, which are the characteristic values. (Note that $b \rightarrow m^{2}$ if $q \rightarrow 0$, and that the eigenfunctions then become $\cos m \theta$ and $\sin m \theta$.)

Since $H_{m}$ is periodic in $\eta$, it may be expressed as a Fourier series of sines and cosines. The solutions, known as Mathieu functions, may be divided into the four different types (e.g., Morse and Feshbach, 1953; Meixner, 1954)

$$
\begin{array}{clc}
c e_{2 m}(\eta, q) & =\sum_{r=0}^{\infty} A_{2 r}^{2 m}(q) \cos 2 r \eta & \text { even in } \eta \\
s e_{2 m+2}(\eta, q) & =\sum_{r=0}^{\infty} B_{2 r}^{(2 m+2)}(q) \sin 2 r \eta & \text { odd in } \eta \\
c e_{2 m+1}(\eta, q) & =\sum_{r=0}^{\infty} A_{2 r+1}^{(2 m+1)}(q) \cos (2 r+1) \eta & \text { even in } \eta \\
s e_{2 m+1}(\eta, q) & =\sum_{r=0}^{\infty} B_{2 r+1}^{(2 m+1)}(q) \sin (2 r+1) \eta & \text { odd in } \eta
\end{array}
$$$$
\text { periodic with period } \pi
$$

Each eigenfunction corresponds to an eigenvalue $b e_{m}$, or $b o_{m}$ (even or odd). Corresponding to the same eigenvalue, we have the solutions of (2.6), which are called modified Mathieu functions

$$
M c_{2 m}^{(1)}, M s_{2 m+2}^{(1)}, M c_{2 m+1}^{(1)}, M s_{2 m+1}^{(1)} \text {. }
$$

As $q \rightarrow 0$ these solutions become Bessel functions $J_{m}$. Also, solutions

$$
M c_{2 m}^{(2)}, M s_{2 m+2}^{(2)}, M c_{2 m+1}^{(2)}, M s_{2 m+1}^{(2)}
$$

become Neumann functions $Y_{m}$ as $q \rightarrow 0$. Linear combinations of the above are also solutions

As $q \rightarrow 0$

$$
\begin{aligned}
& M c_{2 m}^{(3)}=M c_{2 m}^{(1)}+i M c_{2 m}^{(2)} \\
& M c_{2 m}^{(4)}=M c_{2 m}^{(1)}-i M c_{2 m}^{(2)} .
\end{aligned}
$$

$$
\begin{aligned}
& M c_{2 m}^{(3)} \rightarrow \text { Hankel function of the first kind and } \\
& M c_{2 m}^{(4)} \rightarrow \text { Hankel function of the second kind. }
\end{aligned}
$$

The above "radial" solutions may be expanded in series of Bessel functions once the coefficients $A_{2 r}^{2 m}(q), B_{2 r+2}^{2 m}(q), A_{2 r+1}^{2 m+1}(q)$ and $B_{2 r+1}^{2 m+1}(q)$ are known. These coefficients may be calculated by a recursion relation, and are also available in tabulated form for limited values of $q$ (Abramowitz and Stegun, 1964; Meixner, 1954). 


\section{The Plane Wave in Elliptical Coordinates}

The plane wave traveling in the direction with its positive normal making an angle $\theta$ with the positive $x$-axis is

$$
u_{z}^{i}=\exp (-i \omega t) \exp [i k(x \cos \theta+y \sin \theta)],
$$

using (1.1) and (1.2), (3.1) can be transformed into the elliptical coordinates

$$
u_{z}^{i}=\exp (-i \omega t) \exp [i k a(\cosh \xi \cos \eta \cos \theta+\sinh \xi \sin \eta \sin \theta)] .
$$

Letting

and since

$$
W=\cosh \xi \cos \eta \cos \theta+\sinh \xi \sin \eta \sin \theta
$$

[from (2.7)], there follows

$$
k a=2 \sqrt{ } q
$$

$$
u_{z}^{i}=\exp (-i \omega t) \exp (i 2 \sqrt{q} W)
$$

Since (3.4) [i.e., (3.2)] is periodic in $\theta$, it can be expanded into a generalized Fourier series of Mathieu functions

$$
u_{z}^{i}=\sum_{m=0}^{\infty}\left[C_{m} c e_{m}(\theta, q)+D_{m} s e_{m}(\theta, q)\right]
$$

where (by orthogonality of these functions)

$$
\begin{aligned}
& C_{m}=\frac{1}{\pi} \int_{0}^{2 \pi} \exp (i 2 \sqrt{q} W) c e_{m}(\theta, q) d \theta \\
& D_{m}=\frac{1}{\pi} \int_{0}^{2 \pi} \exp (i 2 \sqrt{q} W) s e_{m}(\theta, q) d \theta .
\end{aligned}
$$

The integrals in (3.6a) and (3.6b) are evaluated as (Abramowitz and Stegun, 1964; Meixner, 1954)

$$
\begin{aligned}
& \int_{0}^{2 \pi} \exp (i 2 \sqrt{q} W) c e_{m}(\theta, q) d \theta=2 \pi i^{m} c e_{m}(\eta, q) M c_{m}^{(1)}(\xi, q) \\
& \int_{0}^{2 \pi} \exp \left(i^{2} \sqrt{q} W\right) s e_{m}(\theta, q) d \theta=2 \pi i^{m} \operatorname{se}_{m}(\eta, q) M s_{m}^{(1)}(\xi, q) .
\end{aligned}
$$

Hence, the plane wave expansion in terms of Mathieu functions becomes

$$
u_{z}^{i}=2 \sum_{m=0}^{\infty} i^{m} c e_{m}(\eta, q) M c_{m}^{(1)}(\xi, q) c e_{m}(\theta, q)+2 \sum_{m=1}^{\infty} i^{m} s e_{m}(\eta, q) M s_{m}^{(1)}(\xi, q) s e_{m}(\theta, q)
$$

\section{The Elastic Inclusion: An Elliptical Valley}

We now proceed to solve the problem of a half-space where the elastic properties inside the elliptically shaped valley, $\xi \leqq \xi_{0}$, are different from those outside $\xi=\xi_{0}$. The solution inside the valley, $u_{z}{ }^{v}$ for $\xi \leqq \xi_{0}$, must satisfy the differential equation

$$
\nabla^{2} u_{z}{ }^{v}+k_{v}{ }^{2} u_{z}^{v}=0
$$

and the boundary conditions

$$
\begin{array}{ll}
\mu_{v} \frac{\partial u_{z}^{v}}{\partial \eta}=0 & \text { at } \eta=0,-\pi \text { and }|x|>a \\
\mu_{v} \frac{\partial u_{z}^{v}}{\partial \xi}=0 & \text { at } \xi=0 \text { and }|x| \leqq a
\end{array}
$$


where $\mu_{v}$ is the rigidity for $\xi \leqq \xi_{0}$. We note that within the focal length, the normal derivative is no longer $\partial / \partial \eta$ but $\partial / \partial \xi$.

The solution outside the elliptical valley, $u_{z}=u_{z}^{i+r}+u_{z}{ }^{R}$, (where $u_{z}{ }^{R}$ represents the wave scattered and diffracted from $\xi=\xi_{0}$, and motion $u_{z}^{i+r}$ is the complete solution of the homogeneous half-space problem for incident $u_{z}{ }^{i}$ waves) must satisfy the differential equation

and the boundary conditions

$$
\nabla^{2} u_{z}+k^{2} u_{z}=0
$$

$$
\begin{array}{cc}
\mu \frac{\partial}{\partial \eta}\left(u_{z}{ }^{R}+u_{z}^{i+r}\right)=0 & \text { at } \eta=0,-\pi \\
u_{z}{ }^{v}=u_{z}{ }^{R}+u_{z}^{i+r} \quad \text { at } \xi=\xi_{0} \\
\mu_{v} \frac{\partial u_{z}{ }^{v}}{\partial \xi}=\mu \frac{\partial}{\partial \xi}\left(u_{z}{ }^{R}+u_{z}^{i+r}\right) & \text { at } \xi=\xi_{0} .
\end{array}
$$

Case A: Shallow Valley. We take the coordinate system as shown in Figure 1 and the half-space defined by $y \leqq 0$. The solution in the homogeneous half-space $u_{z}^{i+r}$ is then obtained by adding the series (3.7) for angle $\theta$ to another series (3.7) for angle $-\theta$. Since $\operatorname{se}_{m}(\theta, q)$ are odd in $\theta$, their contribution cancels out and there follows

$$
\begin{aligned}
u_{z}^{i+r} & =4 \sum_{m=0}^{\infty}(-1)^{m} c e_{2 m}(\eta, q) M c_{2 m}^{(1)}(\xi, q) c e_{2 m}(\theta, q) \\
& +4 \sum_{m=0}^{\infty}(-1)^{m} i c e_{2 m+1}(\eta, q) M c_{2 m+1}^{(1)}(\xi, q) c e_{2 m+1}(\theta, q) .
\end{aligned}
$$

The solution $u_{z}{ }^{R}$, the wave reflected from the valley, may be taken to have the form

$$
u_{z}^{R}=\sum_{m=0}^{\infty}\left[a_{m} M c_{m}^{(3)}(\xi, q) c e_{m}(\eta, q)+b_{m} M s_{m}^{(3)}(\xi, q) s e_{m}(\eta, q)\right]
$$

since $M c_{m}^{(3)}(\xi, q)$ and $M s_{m}^{(3)}(\xi, q)$ are solutions corresponding to the outward propagating waves. Since $u_{z}^{i+r}$ already satisfies the zero-stress condition at the surface $y=0$, we now require that

Since,

$$
\mu \frac{\partial}{\partial \eta}\left(u_{z}^{R}\right)=0 \quad \text { at } \eta=0,-\pi .
$$

and

$$
\text { only } \frac{d}{d \eta} c e_{m}(\eta, q)=0 \quad \text { at } \eta=0,-\pi
$$

$$
\frac{d}{d \eta} s e_{m}(\eta, q) \neq 0 \quad \text { at } \eta=0,-\pi
$$

the solution $u_{z}{ }^{R}$ must have the form

$$
u_{z}^{R}=\sum_{m=0}^{\infty}\left[a_{2 m} M c_{2 m}^{(3)}(\xi, q) c e_{2 m}(\eta, q)+a_{2 m+1} M c_{2 m+1}^{(3)}(\xi, q) c e_{2 m+1}(\eta, q)\right]
$$

The solution inside the elliptical valley may be written as

where

$$
\begin{aligned}
u_{z}^{v}= & \sum_{m=0}^{\infty}\left[a_{m} M c_{m}^{(2)}\left(\xi, q^{*}\right)+c_{m} M c_{m}^{(1)}\left(\xi, q^{*}\right) c e_{m}\left(\eta, q^{*}\right)\right. \\
& \left.+f_{m} M c_{m}^{(2)}\left(\xi, q^{*}\right)+e_{m} M s_{m}^{(1)}\left(\xi, q^{*}\right) s e_{m}\left(\eta, q^{*}\right)\right]
\end{aligned}
$$

$$
q^{*}=\left(a^{2} \omega^{2} / 4 \beta_{v}{ }^{2}\right)
$$


All terms with $\operatorname{se}_{m}\left(\eta, q^{*}\right)$ drop out because $(d / d \eta) s e_{m}\left(\eta, q^{*}\right) \neq 0$ at $\eta=0,-\pi$. Although the function $M c_{m}^{(2)}$ is "finite" at the focal length but its derivative is nonzero, i.e.,

therefore

$$
(d / d \xi) M c_{m}^{2}\left(\xi, q^{*}\right) \neq 0 \quad \text { at } \xi=0
$$

$$
u_{z}^{v}=\sum_{m=0}^{\infty} c_{m} M c_{m}^{(1)}\left(\xi, q^{*}\right) c e_{m}\left(\eta, q^{*}\right)
$$

Separating (4.11) into functions with period $2 \pi$ and $\pi$ yields,

$$
u_{z}^{v}=\sum_{m=0}^{\infty}\left[c_{2 m} M c_{2 m}^{(1)}\left(\xi, q^{*}\right) c e_{2 m}\left(\eta, q^{*}\right)+c_{2 m+1} M c_{2 m+1}^{(1)}\left(\xi, q^{*}\right) c e_{2 m+1}\left(\eta, q^{*}\right)\right] .
$$

The displacements $u_{z}^{i+r}+u_{z}{ }^{r}$ and $u_{z}{ }^{v}$ must satisfy the boundary conditions (4.5) and (4.6) by which the coefficients $a_{2 m}, a_{2 m+1}, c_{2 m}$, and $c_{2 m+1}$ may be determined. The first condition (4.5) requires

$$
\begin{aligned}
\sum_{m=0}^{\infty}[ & c_{2 m} M c_{2 m}^{(1)}\left(\xi_{0}, q^{*}\right) c e_{2 m}\left(\eta, q^{*}\right)+c_{2 m+1} M c_{2 m+1}^{(1)}\left(\xi_{0}, q^{*}\right) c e_{2 m+1}\left(\eta, q^{*}\right) \\
= & \sum_{m=0}^{\infty}\left[a_{2 m} M c_{2 m}^{(3)}\left(\xi_{0}, q\right) c e_{2 m}(\eta, q)+a_{2 m+1} M c_{2 m+1}^{(3)}\left(\xi_{0}, q^{*}\right) c e_{2 m+1}(\eta, q)\right] \\
& +\sum_{m=0}^{\infty}\left[4(-1)^{m} c e_{2 m}(\eta, q) M c_{2 m}^{(1)}\left(\xi_{0}, q\right) c e_{2 m}(\theta, q)\right. \\
& \left.+4(-1)^{m} i c e_{2 m+1}(\eta, q) M c_{2 m+1}^{(1)}\left(\xi_{0}, q\right) c e_{2 m+1}(\theta, q)\right] .
\end{aligned}
$$

Similarly condition (4.6) requires

$$
\begin{aligned}
\mu_{v} & \sum_{m=0}^{\infty}\left[c_{2 m} M c_{2 m}^{(1)}\left(\xi_{0}, q^{*}\right) c e_{2 m}\left(\eta, q^{*}\right)+c_{2 m+1} M c_{2 m+1}^{(1)}\left(\xi_{0}, q^{*}\right) c e_{2 m+1}\left(\eta, q^{*}\right)\right] \\
= & \mu \sum_{m=0}^{\infty}\left[a_{2 m} M c_{2 m}^{(3)}\left(\xi_{0}, q\right) c e_{2 m}(\eta, q)+a_{2 m+1} M c_{2 m+1}^{(3)}\left(\xi_{0}, q\right) c e_{2 m+1}(\eta, q)\right] \\
& +\mu \sum_{m=0}^{\infty}\left[4(-1)^{m} c e_{2 m}(\eta, q) M c_{2 m}^{(1)}\left(\xi_{0}, q\right) c e_{2 m}(\theta, q)\right. \\
& \left.+4(-1)^{m} i c e_{2 m+1}(\eta, q) M c_{2 m+1}^{(1)}\left(\xi_{0}, q\right) c e_{2 m+1}(\theta, q)\right] .
\end{aligned}
$$

Here "," designates the derivative of a function with respect to $\xi$. Since $q$ and $q^{*}$ are not the same for both media, i.e.,

$$
q=a^{2} \omega^{2} / 4 \beta^{2} \text { and } q^{*}=a^{2} \omega^{2} / 4 \beta_{v}{ }^{2},
$$

and the Mathieu functions with different values of $q$ are not orthogonal to each other, the coefficients $a_{m}$ and $c_{m}$ may not be separated term by term, rather, infinite matrices result from the expansion of $c e_{2 m}\left(\eta, q^{*}\right)$ and $c e_{2 m}(\eta, q)$ into the Fourier series

and

$$
\begin{aligned}
c e_{2 m}\left(\eta, q^{*}\right) & =\sum_{n=0}^{\infty} A_{2 n}^{2 m *} \cos 2 n \eta \\
c e_{2 m}(\eta, q) & =\sum_{n=0}^{\infty} A_{2 n}^{2 m} \cos 2 n \eta
\end{aligned}
$$

$$
\begin{aligned}
c e_{2 m+1}\left(\eta, q^{*}\right) & =\sum_{n=0}^{\infty} A_{2 n+1}^{2 m+1 *} \cos (2 n+1) \eta \\
c e_{2 m+1}(\eta, q) & =\sum_{n=0}^{\infty} A_{2 n+1}^{2 m+1} \cos (24+1) \eta
\end{aligned}
$$


These matrices are

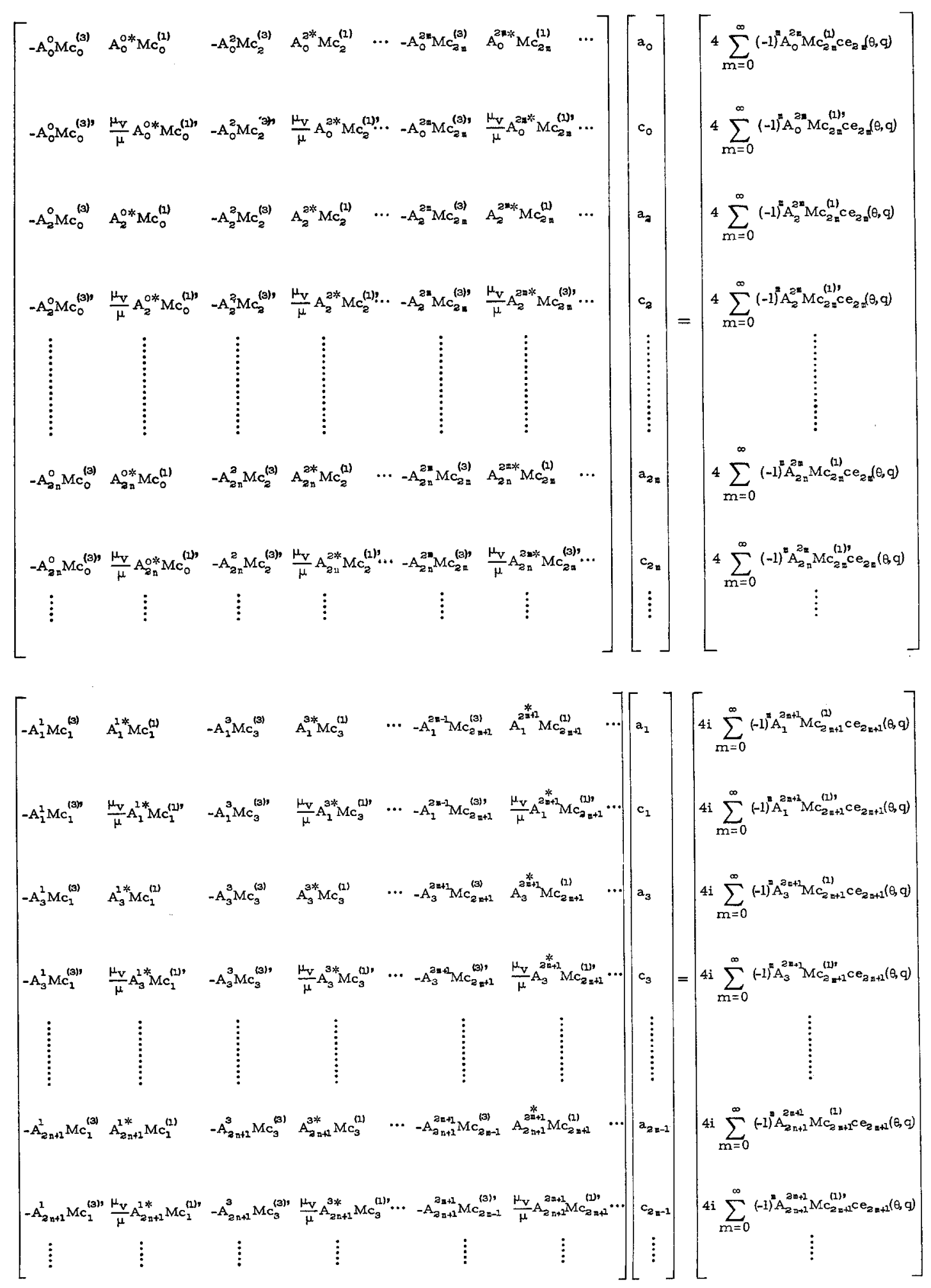


Case B: Deep Valley. For this case, it is convenient to redefine the half-space to be $x \geqq 0$ as in Figure 1 . The formulation of the problem remains the same, but the boundary condition (4.2) is changed to

$$
\mu_{v} \frac{\partial u_{z}^{v}}{\partial \eta}=0 \quad \text { at } \eta= \pm \frac{\pi}{2}
$$

and the boundary condition (4.4) becomes

$$
\mu_{v} \frac{\partial}{\partial \eta}\left(u_{z}^{R}+u_{z}^{i+r}\right)=0 \quad \text { at } \eta= \pm \frac{\pi}{2} .
$$

All other boundary conditions remain unchanged. The half-space solution for this case, $u_{z}^{i+r}$, is obtained by adding the series (3.7) for $\theta$ to the series for $\pi-\theta$.

Since

$$
c e_{2 m+1}(\pi-\theta, q)=-c e_{2 m+1}(\theta, q)
$$

and

$$
s e_{2 m}(\pi-\theta, q)=-s e_{2 m}(\theta, q),
$$

all the terms with $c e_{2 m+1}$ and $s e_{2 m}$ drop out and the solution becomes

$$
\begin{aligned}
u_{z}^{i+r}=4 \sum_{m=0}^{\infty}(-1)^{m} c e_{2 m}(\eta, q) M c_{2 m}^{(1)}(\xi, q) c e_{2 m}(\theta, q) \\
+4 \sum_{m=0}^{\infty}(-1)^{m} i \operatorname{se}_{2 m}(\eta, q) M s_{2 m}^{(1)}(\xi, q) s e_{2 m}(\theta, q)
\end{aligned}
$$

Also, since

$$
\begin{array}{lc}
\frac{d}{d \eta} c e_{2 m+1}(\eta, q) \neq 0 & \text { at } \eta= \pm \frac{\pi}{2} \\
\frac{d}{d \eta} \operatorname{se}_{2 m}(\eta, q) \neq 0 & \eta= \pm \frac{\pi}{2}
\end{array}
$$

the wave scattered from and diffracted around the valley, $u_{z}{ }^{R}$, becomes

$$
u_{z}^{R}=\sum_{m=0}^{\infty}\left[a_{2 m} M c_{2 m}^{(3)}(\xi, q) c e_{2 m}(\eta, q)+a_{2 m+1} M s_{2 m+1}^{(3)}(\xi, q) s e_{2 m+1}(\eta, q)\right]
$$

The solution inside the valley is

$$
u_{z}^{v}=\sum_{m=0}^{\infty}\left[c_{2 m} M c_{2 m}^{(1)}\left(\xi, q^{*}\right) c e_{2 m}\left(\eta, q^{*}\right)+c_{2 m+1} M s_{2 m+1}^{(1)}\left(\xi, q^{*}\right) s e_{2 m+1}\left(\eta, q^{*}\right)\right]
$$

The boundary condition (4.5) now gives

$$
\begin{aligned}
& \sum_{m=0}^{\infty}\left[c_{2 m} M c_{2 m}^{(1)}\left(\xi_{0}, q^{*}\right) c e_{2 m}\left(\eta, q^{*}\right)+c_{2 m+1} M s_{2 m+1}^{(1)}\left(\xi_{0}, q^{*}\right) s e_{2 m+1}\left(\eta, q^{*}\right)\right] \\
= & \sum_{m=0}^{\infty}\left[a_{2 m} M c_{2 m}^{(3)}\left(\xi_{0}, q\right) c e_{2 m}(\eta, q)+a_{2 m+1} M s_{2 m+1}^{(3)}\left(\xi_{0}, q\right) s e_{2 m+1}(\eta, q)\right] \\
+ & \sum_{m=0}^{\infty}\left[4(-1)^{m} c e_{2 m}(\eta, q) M c_{2 m}^{(1)}\left(\xi_{0}, q\right) c e_{2 m}(\theta, q)\right. \\
& \left.+4(-)^{m} i s e_{2 m+1}(\eta, q) M s_{2 m+1}^{(1)}\left(\xi_{0}, q\right) s e_{2 m+1}(\theta, q)\right]
\end{aligned}
$$


while the boundary condition (4.6) gives

$$
\begin{aligned}
\mu_{v} \sum_{m=0}^{\infty}\left[c_{2 m} M c_{2 m}^{(1)}\left(\xi_{0}, q^{*}\right) c e_{2 m}\left(\eta, q^{*}\right)+c_{2 m+1} M s_{2 m+1}^{(1)} s e_{2 m+1}\left(\eta, q^{*}\right)\right] \\
=\mu \sum_{m=0}^{\infty}\left[a_{2 m} M c_{2 m}^{(3)}\left(\xi_{0}, q\right) c e_{2 m}(\eta, q)+a_{2 m+1} M s_{2 m+1}^{(3)} s e_{2 m+1}(\eta, q)\right] \\
+\mu \sum_{m=0}^{\infty}\left[4(-1)^{m} c e_{2 m}(\eta, q) M c_{2 m}^{(1)}\left(\xi_{0}, q\right) c e_{2 m}(\theta, q)\right. \\
\left.+4(-1)^{m} i s e_{2 m+1}(\eta, q) M s_{2 m+1}^{(1)},\left(\xi_{0}, q\right) s e_{2 m+1}(\theta, q)\right]
\end{aligned}
$$

Since the functions $s e_{2 m+1}$ may be expressed as

$$
\begin{aligned}
s e_{2 m+1}\left(\eta, q^{*}\right) & =\sum_{m=0}^{\infty} B_{2 n+1}^{2 m+1^{*}} \sin (2 n+1) \eta \\
s e_{2 m+1}(\eta, q) & =\sum_{n=0}^{\infty} B_{2 n+1}^{2 m+1} \sin (2 n+1) \eta,
\end{aligned}
$$

the infinite matrices have the same form as before except that

$$
\begin{aligned}
& A_{2 n+1}^{2 m+1} \text { becomes } B_{2 n+1}^{2 m+1} \\
& A_{2 n+1}^{2 m+1^{*}} \text { becomes } B_{2 n+1}^{2 m+1 *}, \\
& M c_{2 m+1}^{(1)} \text { becomes } M s_{2 m+1}^{(1)} \text { (same for the derivatives) } \\
& M c_{2 m+1}^{(3)} \text { becomes } M s_{2 m+1}^{(3)} \text { (same for the derivatives) } \\
& c e_{2 m+1} \text { becomes } s e_{2 m+1}
\end{aligned}
$$

These changes, of course, occur only in the second matrix, the first matrix remaining unaltered.

\section{Calculation of the Coefficients}

It is numerically impossible to invert an infinite matrix and a possible alternative is to approximate it by one with finite dimensions. Fortunately, the coefficients $A_{2 n+1}^{2 m+1}, A_{2 n}^{2 m}$, $B_{2 n+1}^{2 m+1}$, etc., are usually the largest and close to 1 if $n \approx m$. For $n=m \pm 3$, the coefficients may be less than $1 / 100$. This indicates that the matrix is "essentially banded," since the diagonal terms are large compared to the off-diagonal terms. This is a favorable situation because it means that $a_{1}$ will not depend much on $a_{5}, a_{7}$, etc., while $a_{5}$ will not depend much on $a_{1}, a_{9}, a_{11}$, etc., so that the penalty for terminating the infinite matrix will not be severe for a range of $q$ that is not too large. It is, of course, wise to calculate more than the number of coefficients necessary and to discard the last few that contribute less than the round-off errors. Excellent results have been obtained by calculating 20 coefficients and discarding the last six.

\section{Surface Displacements}

From the earthquake engineering and strong-motion seismology viewpoints, it is useful to examine the amplitudes and phases describing the motion at various points on the surface of the half-space. Since we have assumed that the excitation consists of an 
infinite train of $S H$ waves with amplitude 1 and frequency $\omega$, these amplitudes and phases are readily available from the solutions (4.7), (4.9), and (4.12) for the shallow valley and from (4.17), (4.18), and (4.19) for the deep valley. For the complex displacements, $u$, we define

$$
\begin{aligned}
\text { amplitude } & \equiv\left\{\operatorname{Re}^{2}(u)+\operatorname{Im}^{2}(u)\right\}^{1 / 2} \\
\text { phase } & =\tan ^{-1}(\operatorname{Im}(u) / \operatorname{Re}(u)) .
\end{aligned}
$$

These amplitudes and phases depend on the frequency and the angle of the incident waves, the ratio of the major to minor axis, $R$, of the semi-elliptical valley, and on the material constants, $\rho, \rho_{v}\left(\rho=\mu / \beta^{2} ; \rho_{v}=\mu_{v} / \beta_{v}^{2}\right), \beta$, and $\beta_{v}$ or on the dimensionless parameters $k A, \rho / \rho_{v}, \beta / \beta_{v}, R$, and $\theta$. The parameter $k A=\omega A / \beta$ is also equal to $2 \pi A / \lambda$. Since $\lambda$ is equal to $\beta T$ it becomes convenient to think in terms of another dimensionless parameter given by ETA $\equiv 2 A / \lambda$ (for the deep valley ETA is equal to $2 A / \lambda R$ ) where $2 A$ is the width of the alluvial valley. This choice of the dimensionless parameter ETA has been motivated by its physical meaning linking the maximum dimension of the alluvial valley with the wavelength of the incident $S H$-waves.

Figures 2 through 5 present the amplitudes and phases plotted versus $x / A$. For simplicity in comparing the different figures and in contrast to the coordinate systems used in derivations of the mathematical solutions, for Figures 5 and 6 we use $x_{1}$ as the horizontal (lying in the half-space boundary) and $y$ as the vertical coordinate. Also for uniformity, in all figures THETA $=0$ corresponds to the horizontal (grazing) incidence, while THETA $=90^{\circ}$ corresponds to the vertical incidence of $S H$ waves. The ratios $\mu_{v} / \mu=0.167$ and $\rho_{v} / \rho=0.667$ used for presenting all the figures correspond to the ratio $\beta / \beta_{v}=2.0$. Figures 2,3 , and 4 present the amplitudes and phases for the shallow elliptical valleys with the minor-to-major axis ratios $R=0.1,0.3$, and 0.7 . Figures 5 and 6 present the same for the deep elliptical valleys and for the minor-to-major axis ratios $R=0.7$ and 0.5 .

As may be seen from Figures 2 through 6, when ETA increases (the incident wavelength $\lambda$ decreases) the influence of the valley on the motion of the surrounding halfspace and the complexity of motion in the valley increase. This is in accord with our expectation that the long waves do not "feel" the geological discontinuities with characteristic size smaller than the wavelength in question, whereas the short waves are sensitive to such irregularities.

For short incident waves, small angles of incidence and for $x / A$ typically less than 1 , significant standing-wave patterns may develop in the valley and for $x / A$ less than -1 , i.e., in the direction from which the incident waves arrive. These waves develop from the interference of the incident plane $S H$-waves and the elliptical waves scattered from and refracted through the discontinuity at $\xi=\xi_{0}$ near $x / A=-1$. The standing wave patterns also develop within the valley because for the grazing and acute angles of incidence the soft to hard jump in the rigidities at $x / A=1$ acts as a barrier which reflects appreciable amounts of the wave energy back into the direction of decreasing $x / A$. These standing waves are further characterized by several points along $x / A$ where the displacement amplitudes are zero or are very small and where the phase experiences a jump of essentially $180^{\circ}$. These jumps in the phase diagrams indicate that at these points the ground motion has essentially a torsional character. More detailed discussion on this type of motion and its possible implication may be found in our paper dealing with the vibrations in the semi-cylindrical alluvial valley (Trifunac, 1971) and therefore will not be repeated here.

The phase of surface displacements is plotted in Figures 2 through 6 relative to the phase at $x / A=0$, arbitrarily set to zero, for convenience in presentation. In the absence 

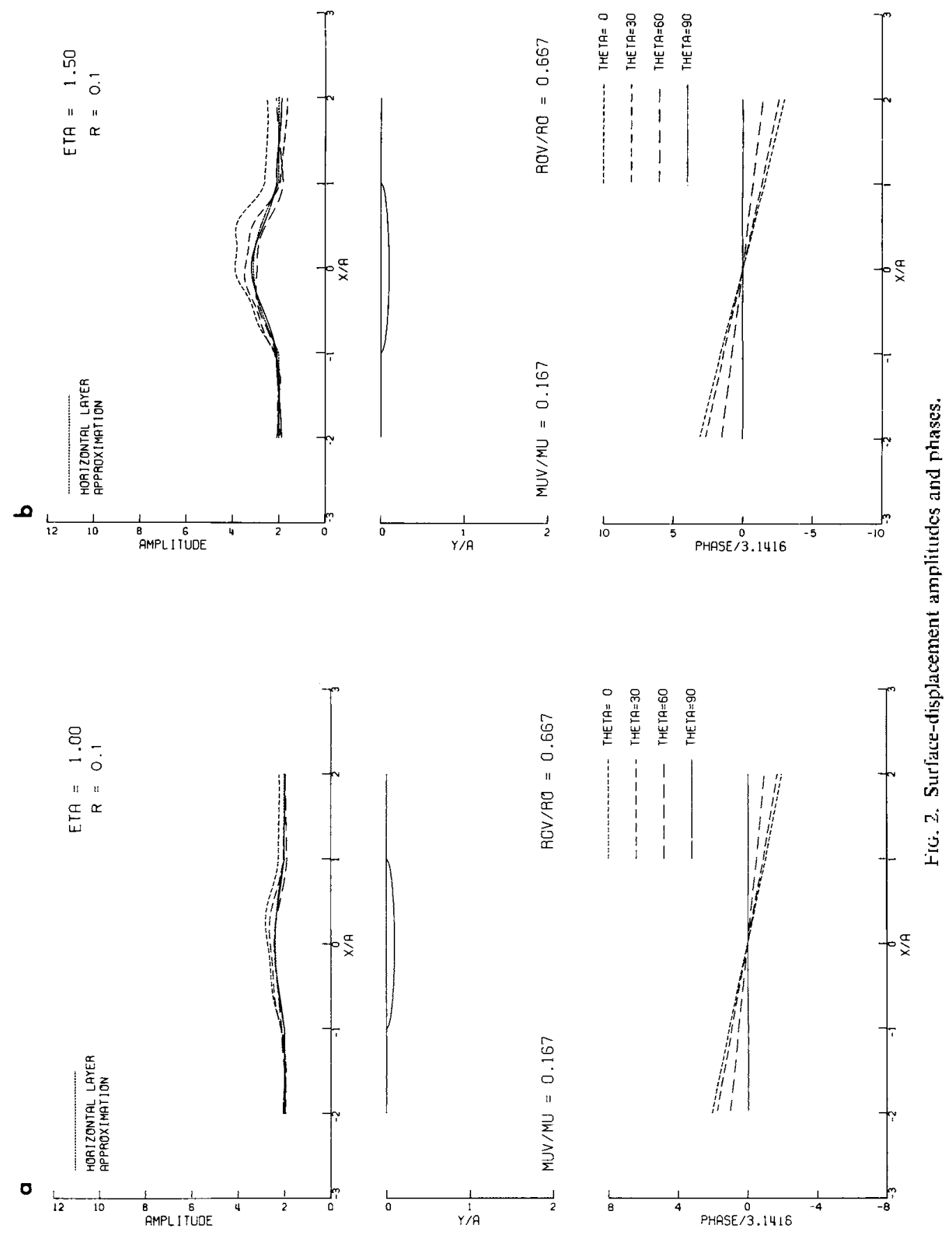

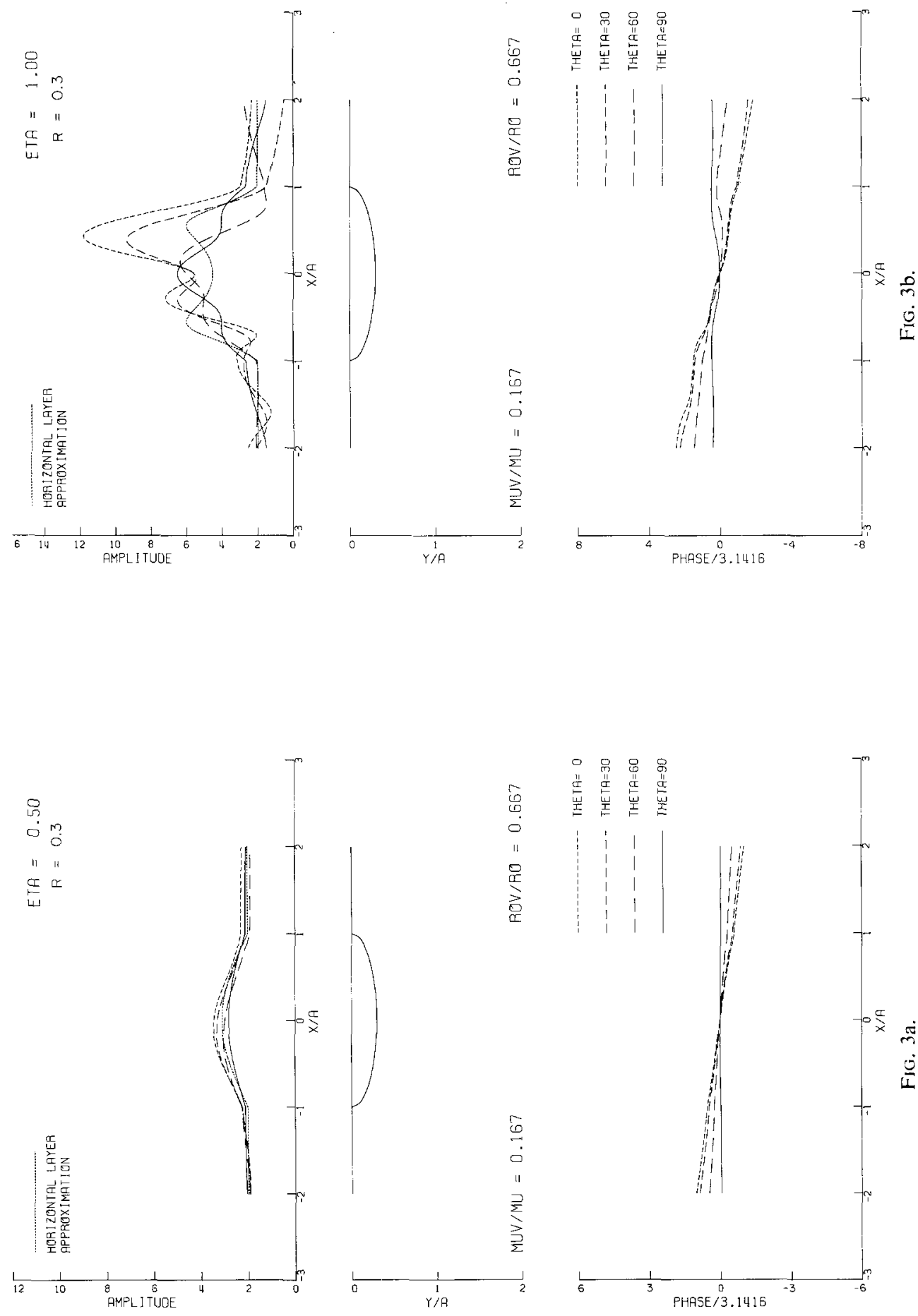

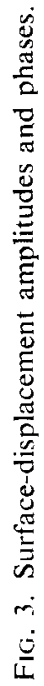


of the alluvial valley this phase would be a straight line

$$
\text { phase }(x / A)=-\frac{\omega A}{c_{x}} \frac{x}{A}=-\frac{\omega A}{\beta} \frac{x}{A} \cos \theta
$$

and its slope would increase for $\theta$ decreasing. Since ETA $=2 A / \lambda$,

$$
\text { phase }(x / A)=-\pi(x / A) \text { ETA } \cos \theta,
$$

and it is seen that with higher ETA the slope of the phase $(x / A)$ increases. The Figures 2 through 6 clearly show these trends in the phase diagrams and demonstrate that the
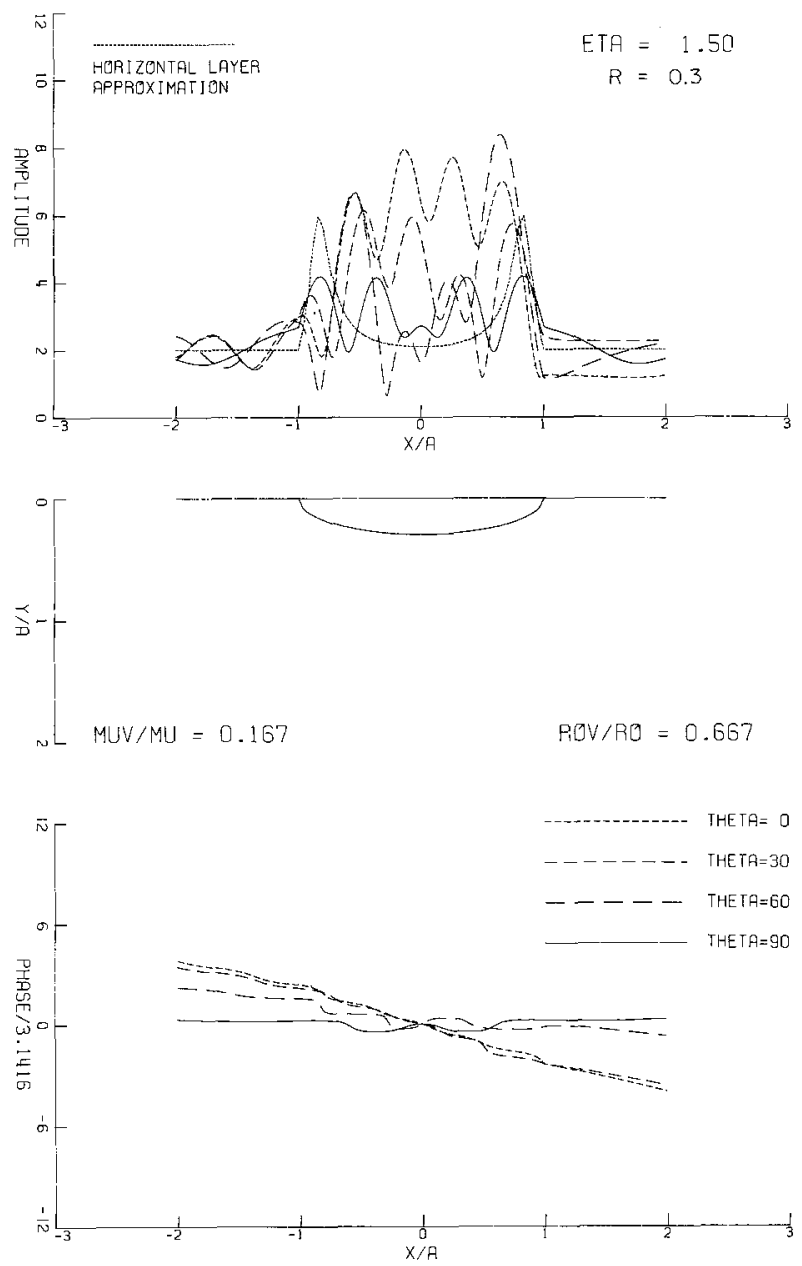

FIG. 3c.

overall nature of the wave propagation is from left to right, as it would be in the absence of the alluvial valley. The local jumps and departures in the phase diagrams from this simple picture are, of course, caused by the interference and scattering of waves in the presence of the alluvial valley.

In engineering practice the approximate model employed in evaluating the influence of local site conditions on the amplification of vertically incident plane $S$ waves consists of a layer of constant thickness $H$. For the material properties $\mu_{v}$ and $\beta_{v}$ of the layer overlying the half-space (characterized by $\mu$ and $\beta$ ) and for the incident waves with frequency 

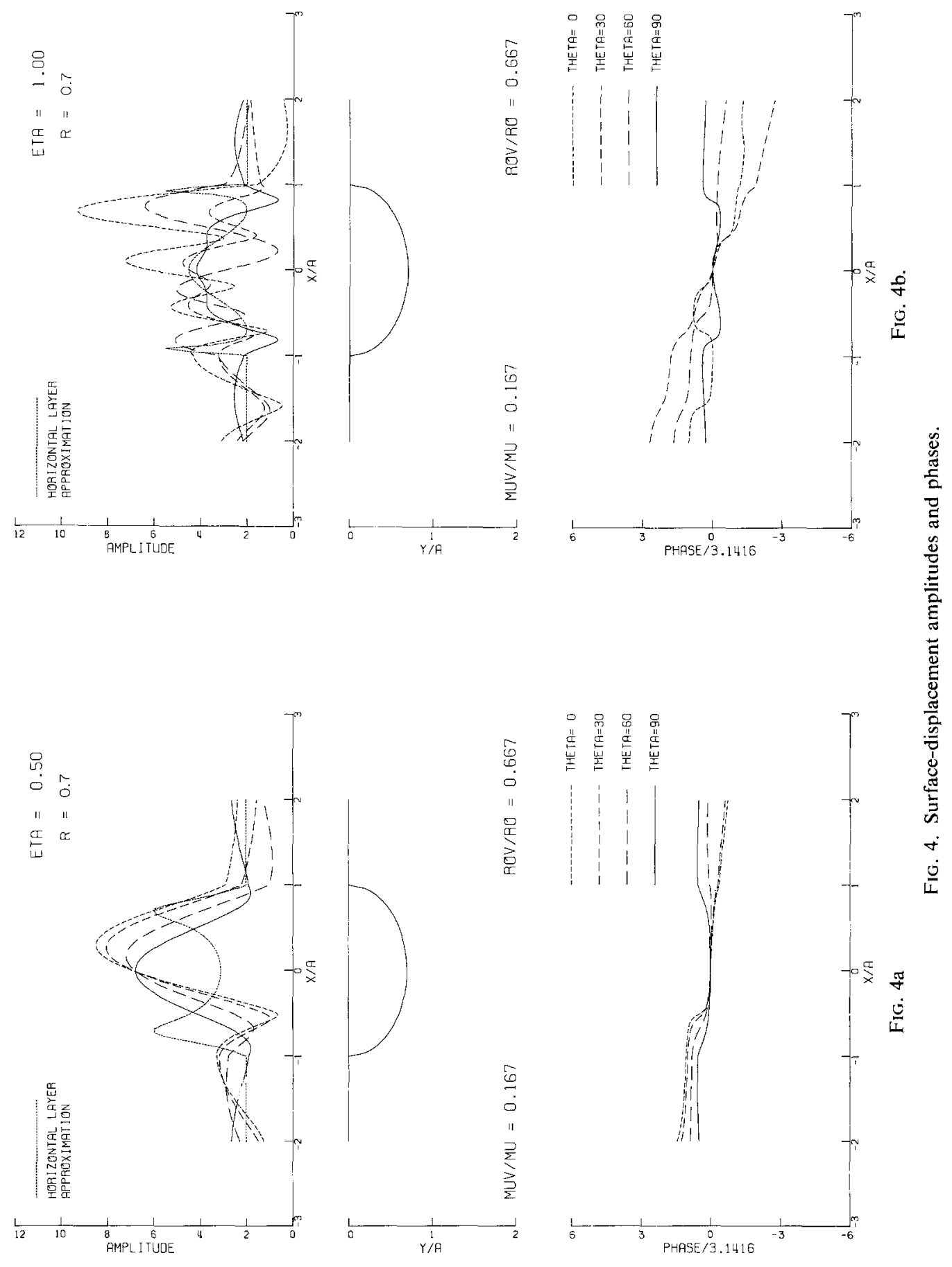
$\omega$ and amplitude equal to 1, the amplitude of surface displacements becomes (Haskell, 1960).

$$
\text { amplitude }=2\left\{\cos ^{2}\left(\omega H / \beta_{v}\right)+\left(\mu_{v} \beta / \mu \beta_{v}\right)^{2} \sin ^{2}\left(\omega H / \beta_{v}\right)\right\}^{-1 / 2}
$$

The maxima of this function occur at $\omega H / \beta_{v}=(2 m+1) \pi / 2$ and are equal to $2 \mu \beta_{v} / \mu_{v} \beta$. To explore the applicability of such a simple approximate model we plotted the above amplitudes versus $x / A$ in Figures 2 through 6 . We note that in different diagrams in Figures 2 through 6 the frequency $\omega$ and $\mu \beta_{v} / \mu_{v} \beta$ are fixed. Only $H$, the depth of the
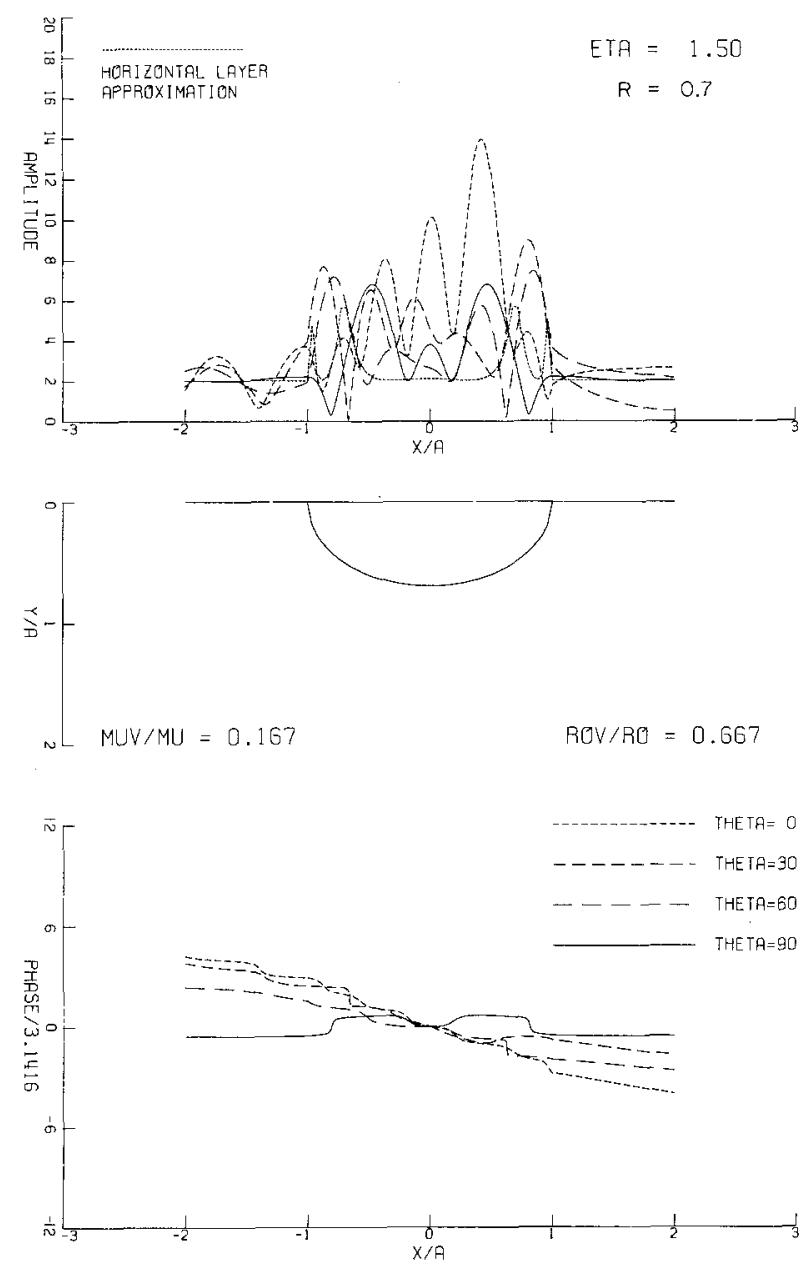

FIG. 4c.

"equivalent" layer, changes with $x / A$ and has been selected to correspond to the depth of the elliptical valley at $x / A$. For $\mu_{v} / \mu=0.167$ and $\rho_{v} / \rho=0.667$ the maximum amplitude of surface motion is 6 . For deeper valleys, when $H$ varies more rapidly with $x / A$ and for larger values of ETA, it will be seen that some of the peaks of the above amplitude appear to be "cut off" and smaller than 6 . This is merely a consequence of the finite number of points used in the computer program that plotted these amplitudes.

Detailed comparison of the surface displacement amplitudes derived from the horizontal layer approximation with those derived from the exact solution in this paper shows that in some limited cases the horizontal layer approximation may lead to good 

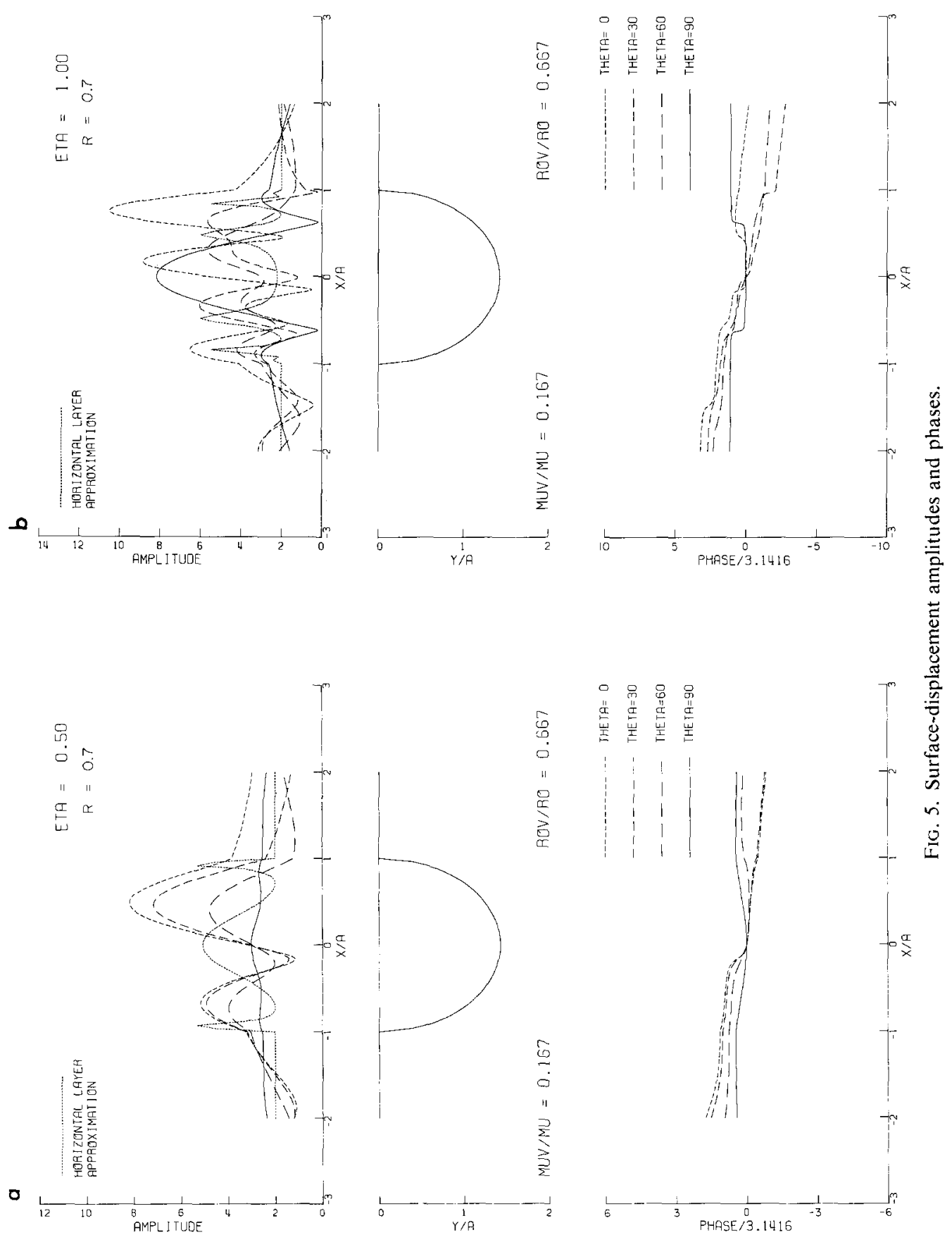
estimates of surface ground displacements. It seems that $\omega H / \beta_{v}$ should be less than $\pi / 2$ for this approximation to hold (Figure 2). For "deeper" alluvial valleys where the local depth exceeds $\beta_{v} \pi / 2 \omega$, the approximate amplification pattern predicted by the horizontal layer model has nothing in common with the exact solution. This disagreement can be explained by the two-dimensional nature of the exact problem displaying horizontal as well as vertical interference patterns, whereas the equivalent horizontal layer model can support only the interference of $S H$ waves in one dimension.
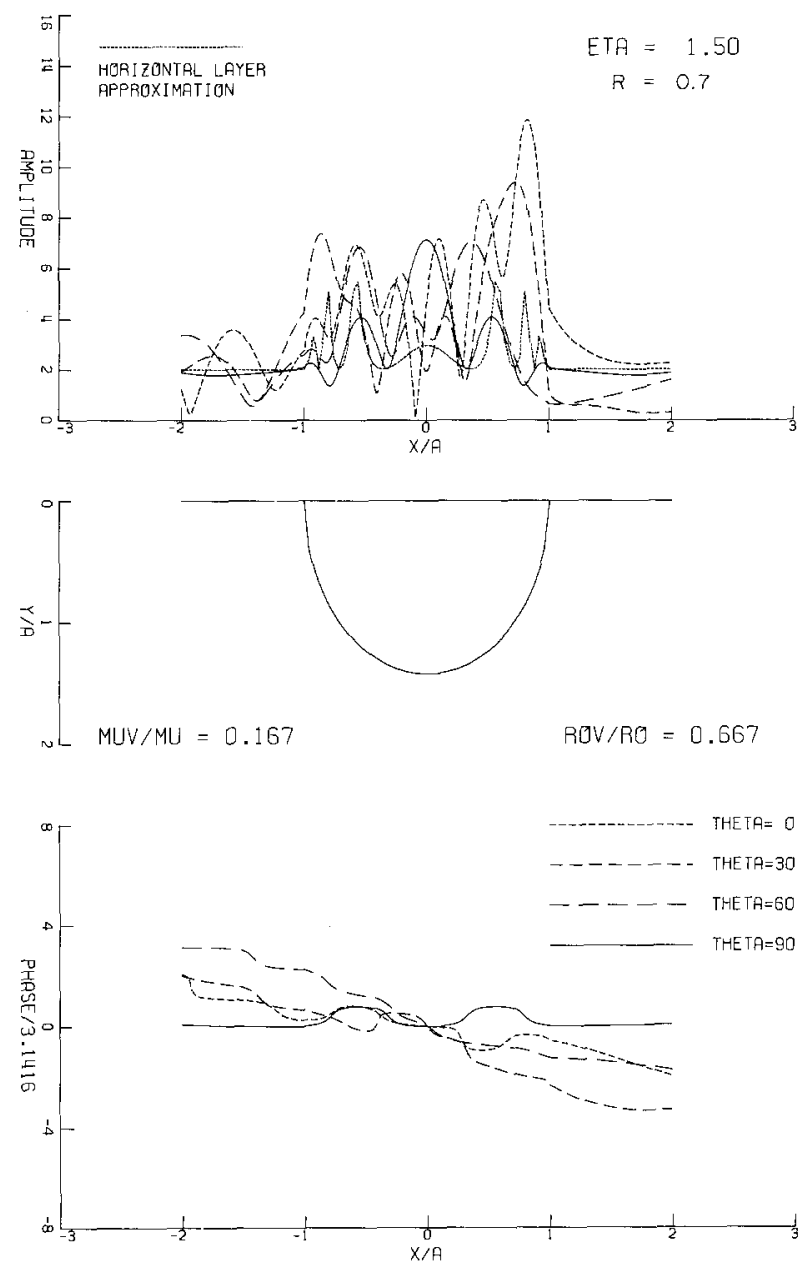

FIG. 5c.

The two-dimensional focusing effects, especially in cases of deep elliptical valleys, lead to large surface amplitudes between $x / A=0$ and $x / A=1$. These amplitudes, often larger than $2 \mu \beta_{v} / \mu_{v} \beta$, are caused by the strong concentration and constructive interference of the waves incident from left and the waves reflected from the discontinuity $\xi=\xi_{0}$ at $x / A=1$.

\section{CONCLUSIONS}

The pattern of surface displacement amplifications for the semi-elliptical alluvial valley has been found to change rapidly over short distances and the amplification accompanying these rapid changes has been found to vary by as much as one order of magnitude. 

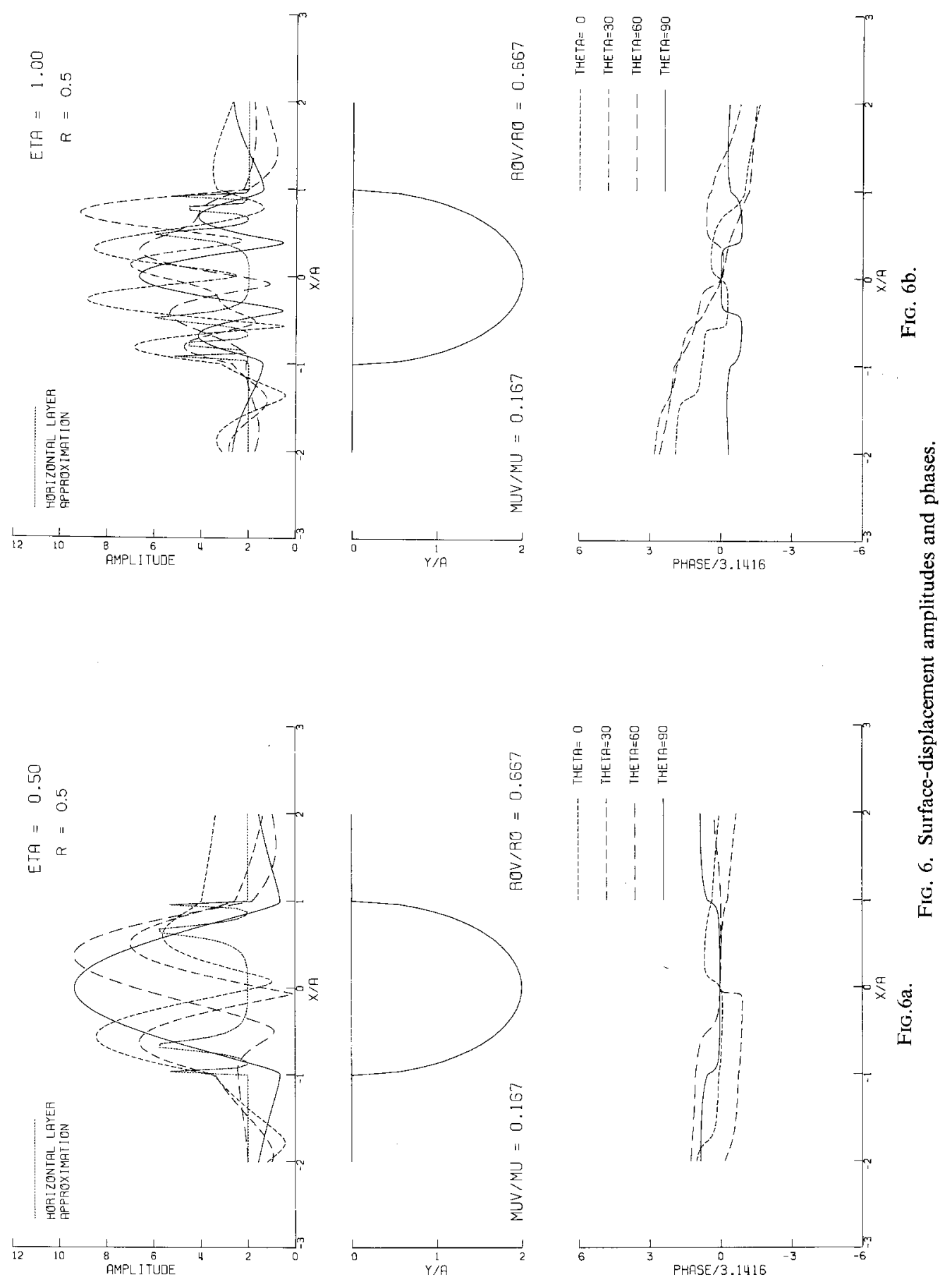
The overall degree of complexity of the amplification pattern and the observed maximum amplitudes both increase with the increasing frequency of the incident plane $S H$-waves. The computed amplification curves for the steady-state excitation seriously depend on the angle of incidence of the $S H$ waves. This dependence is reflected in the change of spectral amplitudes, complete disappearance and occurrence of local peaks, and the change in overall trends of the displacement amplitudes as $\theta$ varies from $0^{\circ}$ to $90^{\circ}$.

A comparison was made of the amplification patterns in the semi-elliptical valley with the amplifications derived from the simple horizontal layer overlying the half-space and excited by the vertically incident $S$-waves. The layer thickness has been selected to correspond to the local depth of the elliptical valley. It has been found that for a limited range of $\omega, \beta_{v}$, and $H$, given by $\omega H / \beta_{v}<\pi / 2$, the two amplification patterns agree for shallow alluvial valleys. For $\omega H / \beta_{v}>\pi / 2$ there is no resemblance between the exact and approximate amplitudes computed from the "equivalent" horizontal later. This conclusion seems to be important for many engineering applications when the explanation of the amplification effects possibly caused by the local soil conditions is attempted by employing the simple horizontal layer overlying the elastic half-space.

\section{ACKNOWLEDGMENTS}

We thank P. C. Jennings and J. E. Luco for critical reading of the manuscript and some useful comments. This research was supported in part by grants from the National Science Foundation and the Earthquake Research Affiliates Program at the California Institute of Technology.

\section{REFERENCES}

Abramowitz, M. and I. A. Stegun (1964). Handbook of Mathematical Functions, National Bureau of Standards.

Aki, K. and K. Larner (1970). Surface motion of a layered medium having an irregular interface due to incident plane $S H$ waves, J. Geophys. Res. 75, 933-954.

Boore, D. (1970). Love waves in nonuniform wave guides: finite difference calculation, J. Geophys. Res. $75,1512-1527$.

Boore, D. M., K. H. Larner and K. Aki (1971). Comparison of two independent methods for the solution of wave-scattering problems: response of a sedimentary basin to vertically incident $S H$ waves, $J$. Geophys. Res. 76, 558-569.

Dezfulian, H. and H. B. Seed (1969). Seismic response of soil deposits underlain by sloping rock boundaries, Report No. EERC 69-9, U. California, Berkeley.

Gutenberg, B. (1957). Effects of ground on earthquake motion, Bull. Seism. Soc. Am. 47, 221-250.

Haskell, N. (1960). Crustal reflection of plane $S H$ waves, J. Geophys. Res. 65, 4147-4150.

Hudson, D. E. (1972). Local distribution of strong earthquake ground motion, Bull. Seism. Soc. Am. 62, $1765-1786$.

Jennings, P. C. (Editor) (1971). San Fernando earthquake of February 9, 1971, Earthquake Eng. Res. Lab., EERL 71-02, Calif. Inst. of Tech., Pasadena.

Meixner, J. (1954). Mathieusche Funktionen und Sphäroidfunktionen, Springer-Verlag, Berlin.

Morse, P. M. and H. Feshbach (1953). Methods of Theoretical Physics, McGraw-Hill, New York.

Mow, C. C. and Y. H. Pao (1971). The diffraction of elastic waves and dynamic stress concentrations, U.S. Air Force Project Pand, $R-482-P R$, April, 1971.

Richter, C. F. (1958). Elementary Seismology, Freeman, San Francisco.

Sozen, M. A., P. C. Jennings, R. B. Matthiesen, G. W. Housner and N. M. Newmark (1968). Engineering Report on the Caracas Earthquake of 29 July 1967, National Academy of Sciences, Washington, D.C.

Tsai, N. C. (1969). Influence of local geology on earthquake ground motion, Earthquake Eng. Res. Lab., Calif. Inst. of Tech., Pasadena.

Trifunac, M. D. (1971). Surface motion of a semi-cylindrical alluvial valley for incident plane $S H$ waves, Bull. Seism. Soc. Am. 61, 1755-1770.

EARThQuake Engineering Research Laboratory

California Institute of Technology

Pasadena, California 91109.

Manuscript received March 18, 1974. 